\title{
Organising pneumonia caused by transtuzumab (Herceptin) therapy for breast cancer
}

\author{
E. Radzikowska, E. Szczepulska, M. Chabowski, I. Bestry
}

Organising pneumonia caused by transtuzumab (Herceptin) therapy for breast cancer. E. Radzikowska, E. Szczepulska, M. Chabowski, I. Bestry. (C) ERS Journals Ltd 2003. ABSTRACT: The case of a 49-yr-old female, who developed fever, effort dyspnoea and cough, with patchy migratory bilateral pulmonary infiltrates 6 weeks after starting transtuzumab therapy, following breast-conserving surgery with adjuvant chemoradiotherapy and hormone therapy for breast carcinoma, is reported here.

Chest radiograph and thin section computed tomography demonstrated alveolar opacities with air bronchogram in both lungs. A lung biopsy was performed in a nonirradiated area of the contralateral lung and revealed a typical histological pattern of organising pneumonia (previously known as bronchiolitis obliterans organising pneumonia). Transtuzumab therapy was discontinued and subsequent gradual clinical and radiological improvement was observed. After 3 months, complete resolution of symptoms and radiographical abnormalities were noted.

This is the first case report that suggests that transtuzumab therapy might induce the development of lung infiltrates with the histological appearance of organising pneumonia.

Eur Respir J 2003; 21: 552-555.
National Tuberculosis and Lung Diseases Research Institute, Warsaw, Poland.

Correspondence: E. Radzikowska, National Tuberculosis and Lung Diseases Research Institute, 26 Plocka Street, 01-138 Warsaw, Poland.

Fax: 48224312408

E-mail: e.radzikowska@igichp.edu.pl

Keywords: Adverse reaction

breast cancer treatment

bronchiolitis obliterans organising

pneumonia

organising pneumonia

transtuzumab

Received: April 302002

Accepted after revision: August 52002
Current treatment for breast cancer is based on a multimodality approach including surgery, chemotherapy, radiotherapy, hormone and biological therapy. Immunotherapy, by humanised anti HER-2 monoclonal antibody against epidermal growth factor receptor 2 (transtuzumab), is one of the biological modes of treatment for breast cancer patients with a high expression of this receptor [1].

During the course of breast cancer treatment, lung damage may occur due to radiotherapy and drugs [2-6]. A wide spectrum of adverse pulmonary reactions, including eosinophilic infiltrates, lipoproteinosis, organising pneumonia (OP) (previously known as bronchiolitis obliterans organising pneumonia (BOOP)), fibrosis and respiratory distress syndrome, may be observed [4-6].

$\mathrm{OP}$ is a nonspecific inflammatory process, which may result from many causes and has been described in association with conditions such as infectious pneumonia, lung abscess, empyema, bronchiectasis, adult respiratory distress syndrome, lung cancer, connective tissue disorders, ulcerative colitis, Crohn's disease, Wegener's granulomatosis, hepatitis C, chronic rejection syndrome, graft versus host disease and mieloproliferative disorders. It may be induced by drugs or radiotherapy. A cryptogenic form of OP may also be observed [4-6]. The disease is pathologically defined by the presence of buds of granulation tissue formed by fibrin exudates, fibroblasts, myofibroblasts and loose connective tissue in distal air spaces (alveolar spaces and bronchiolar lumen), although lung structure is preserved $[5,6]$.

OP is characterised by flu-like symptoms with temperature, cough, dyspnoea, crackles and patchy, often migratory, infiltrates on chest roentgenograms $[5,6]$. These types of changes have been previously described in breast cancer patients undergoing radiotherapy but no cases of OP in the course of transtuzumab therapy have been presented $[2,3,7]$.

This report documents the first case of OP connected with transtuzumab therapy and its spontaneous remission after the treatment was discontinued.

\section{Case report}

A 49-yr-old nonsmoking female, epileptic since childhood (treated for several years previously with lamotrigine), underwent breast-conserving surgery in June 2001 for adenocarcinoma of the left breast. The pathological findings revealed ductal and intraductal cancer infiltration, which formed structures of comedocarcinoma type. Examination demonstrated neoplastic cells in 16 out of 22 axillary lymph nodes. Moreover, immunohistochemical staining of the neoplastic cells displayed oestrogen $(2+)$, progesterone $(3+)$ and HER-2 $(3+)$ receptors. Considering the extent of the neoplasm, adjuvant treatment was administered, including letrozol and four courses of chemotherapy (adriamycine and cyclophosphamide). Then, from October 30 to December 02 2000, radiotherapy of 4,250 cGy was applied on the area comprising the supraclavicular lymph nodes, the axillary lymph nodes and the left breast (17 fractions for 24 days, a boost of 1,600 cGy on the postresectional area and 1,000 cGy in five fractions on the lower and central level of the left axilla). Having finished radiotherapy, chemotherapy of docetaxel (four courses) was given every 3 weeks. Since January 22 2001, infusions of transtuzumab were added every week, starting from the loading dose. During the first infusion the patient developed fever $\left(38^{\circ} \mathrm{C}\right)$, which disappeared when paracetamol was applied. After 6 weeks of transtuzumab treatment she experienced subfebrile status, weakness, decreased exertion tolerance and a dry cough. The cefuroxim and norfloxacin treatment for suspicion of a respiratory tract infection was initiated, but without any effect. The patient was not recovering. On April 202001 she was 
hospitalised with symptoms of bilateral pneumonia, treated with ceftazidim and transtuzumab treatment was continued.

The patient was admitted to the National Tuberculosis and Lung Diseases Research Institute in May 2001. On admission she was alert, without loss of body mass. The patient complained of weakness, effort dyspnoea, nonproductive cough and a low-grade fever. On auscultation, the breath sounds were diminished at the base of both lungs and wheezes were heard at the base of the left lung. The laboratory tests revealed an erythrocyte sedimentation rate (ESR) elevated to $57 \mathrm{~mm} \cdot \mathrm{h}^{-1}$. Tests for antinuclear antibodies, rheumatic factor and $\mathrm{Myco-}$ plasma pneumoniae antibodies were negative. A chest radiograph showed patchy nonsegmental bilateral areas of air space consolidation in the right lower lobe as well as in the left apex and the lingula (fig. 1). The variability of radiograph examinations was observed as the infiltrates in the right lung and in the left middle lobe slightly diminished and the infiltrates in the left upper lobe increased. The high-resolution computed tomography scan disclosed patchy bilateral foci of air space consolidation, mainly in subpleural regions, mild bronchial wall thickening and dilatation within areas of "ground glass" opacities at the base of the right lung and at the apex and lingula of the left lung (fig. 2). The bronchoscopic evaluation showed a normal bronchial tree without any signs of inflammation. The microbiological and cytological examinations of bronchial secretion were negative. The spirometric tests did not reveal any ventilatory disorders; vital capacity (VC) $3.31 \mathrm{~L}(100 \%)$, forced expiratory volume in one second (FEV1) 2.831 (101\%), FEV1 85\% of 100\% VC (107\% of normal) and carbon monoxide transfer coefficient were normal. An arterial oxygen tension of $11.70 \mathrm{kPa}(88 \mathrm{mmHg})$ and a carbon dioxide arterial tension of $5.32 \mathrm{kPa}(40 \mathrm{mmHg})$ were determined.

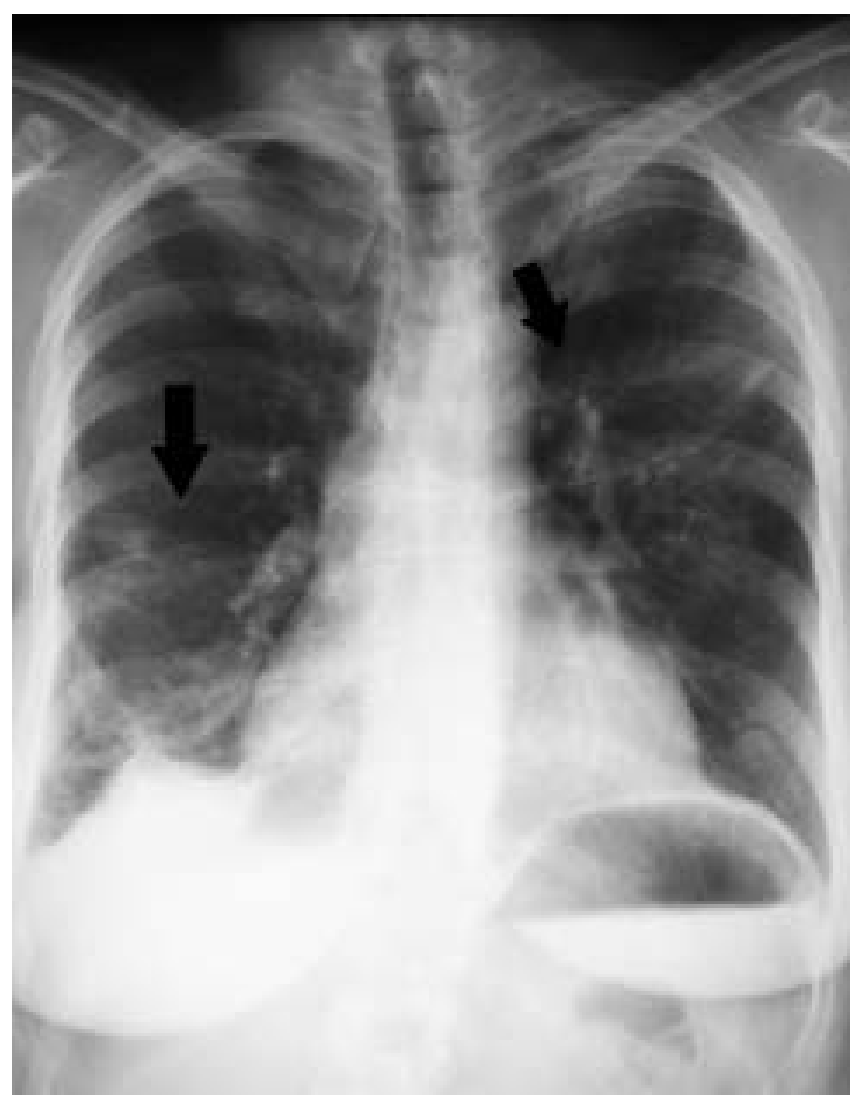

Fig. 1.-Chest radiograph showing patchy nonsegmental bilateral areas of air space consolidation in the left apex in the lingula and in the right lower lobe (arrows).
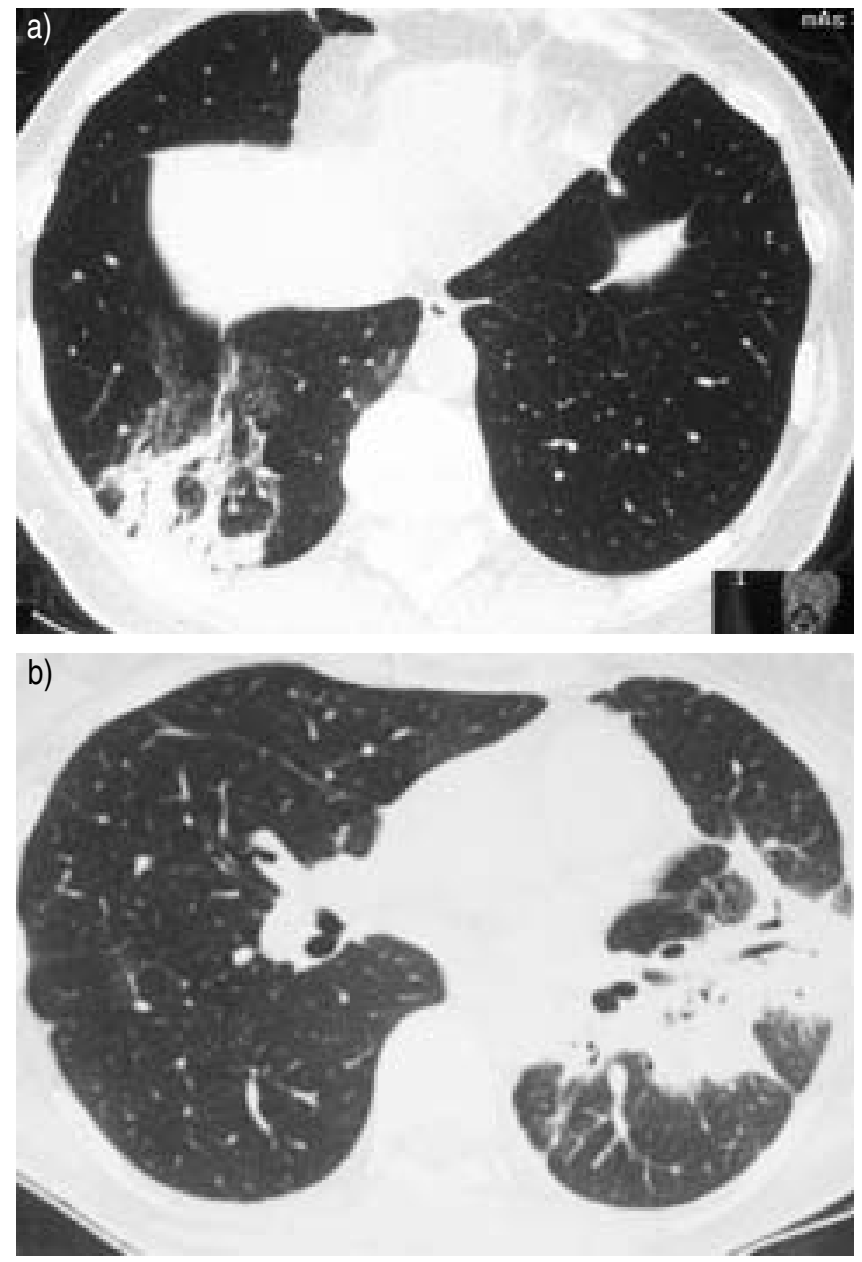

Fig. 2. - Thin section computed tomography slice through the lower lung zones. Patchy foci of airspace consolidation, ground glass opacities and bronchial dilatation are present throughout the basilar segments of the a) right and b) left lower lobe.

An open lung biopsy was performed and specimens of the right lower and middle lobe were obtained. Copious inflammation with thickened intra-alveolar septa and the inflammatory infiltration of mononuclear cells (the areas of a nonspecific interstitial inflammation) were demonstrated. Moreover, the BOOP-like areas of buds of granulation tissue and loose connective tissue in bronchiolar lumen, gigantic cell granuloma with fissures caused by diluted cholesterol crystals and the areas of the intra-alveolar exudates were revealed (fig. 3). Biopsy specimens were negative for pathogens. One week before lung biopsy, transtuzumab administration was stopped as it was suspected that disease might be caused by this drug. The treatment of letrozol was continued. One week after surgical procedure a slight regression of lung opacities on chest radiograph was observed, therefore steroid therapy was postponed. The patient made a gradual recovery and the control chest radiograph showed a marked regression. Three months after withdrawal of transtuzumab, near total regression of the pulmonary densities was noted on the radiograph, except for small reticular changes in the left upper lobe (fig. 4). Ventilatory values were in the normal limits (VC: $3.1 \mathrm{~L}, 94 \%$ of normal; FEV1: 2,751, 97\% of normal).

\section{Discussion}

Two points should be discussed for this case, the first generally concerning OP and the second concerning OP development in the course of transtuzumab therapy. 


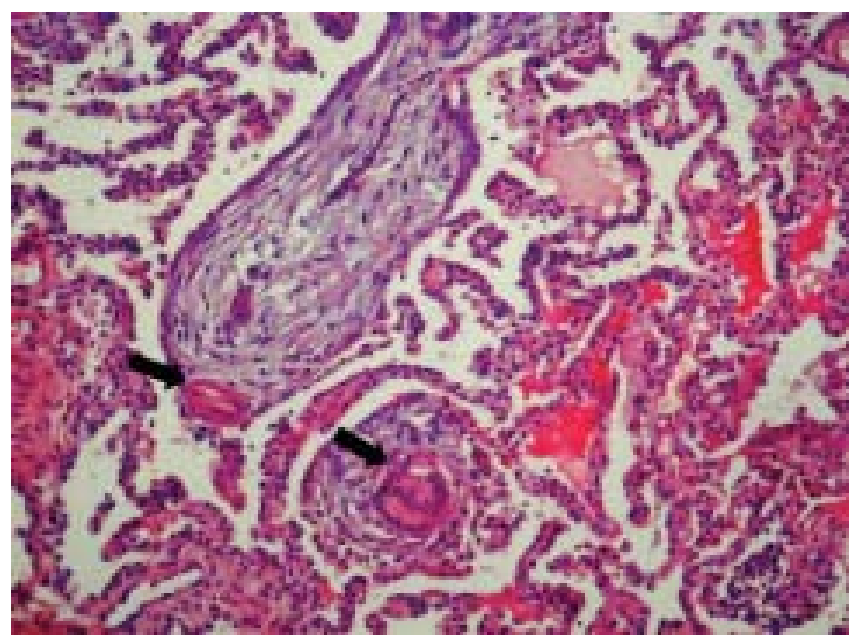

Fig. 3.-Open lung biopsy specimen showing an inflammation with thickened interalveolar septa and the inflammatory infiltration of mononuclear cells (areas of a nonspecific interstitial inflammation). Areas of buds of granulation tissue and loose connective tissue in alveolar and bronchiolar lumen, gigantic cell with fissures caused by diluted cholesterol crystals (arrows) and the areas of the intra-alveolar exudates are shown. Haematoxylin and eosin stain.

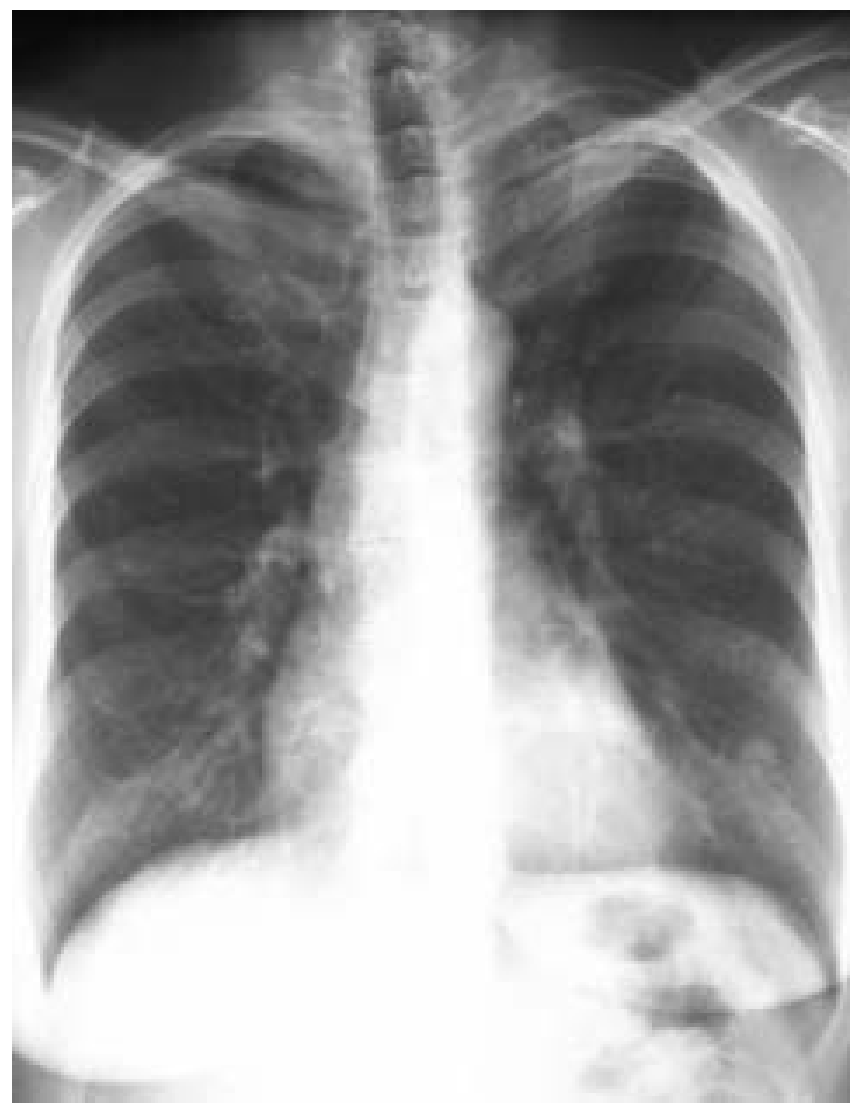

Fig. 4. - Chest radiograph showing regression of pulmonary changes despite the small reticular opacities in the apex of the left lung.

The pathological findings of OP are not specific for any condition. It is noted as an effect of post-drug reactions (amiodaron, nitrofurantoin, acebutolol, amphotericine, cefradin, cocaine, gold-derivate, penicylamine, sulfasalazine, sulindac, phenytoin, vianbarbital), connected with injury to the lung caused by chemical substances and may be idiopathic [4-6]. This disease can also be observed in patients treated for neoplasm after bone marrow transplantation, bleomycin, busulfan, cyclophosphamide, metothrexate, mitomycin-C, interferon $2 \alpha$ and $\beta 1$ a treatment and during radiotherapy [3-5]. However, to the best of the authors' knowledge, no other cause of OP was identified during the course of anti-oestrogen treatment. The authors believe that in this case pulmonary densities were not connected with radiotherapy. Despite their manifestation 3 months following radiotherapy (changes were frequently observed 2-18 months after radiotherapy), described opacities did not tend to spontaneously regress and required intensive steroid therapy, often being recurrent after its withdrawal. Other causes of OP were excluded. There was no clinical and serological evidence of collagen-vascular disease, no exposure to fumes, toxins, a negative search for pathogens in bronchoalveolar lavage and biopsy specimens, and negative serological testing for Mycoplasma pneumoniae. Pulmonary infiltrates tended to migrate and antibiotic therapy did not influence the course of disease. The radiological findings, particularly those shown in high-resolution computed tomography, with bilateral pulmonary infiltrates of different size and localisation, suggested OP. Histological investigation of pulmonary tissue is decisive for making a diagnosis [6]. An open lung biopsy was performed in this case and the histological findings were typical of OP (buds of granulation tissue in alveoli and alveolar ducts, the infiltration of alveolar walls by mononuclear cells, alveolar space foam cells and the absence of interstitial fibrosis). Gigantic cells with fissures caused by diluted cholesterol crystals were also shown in biopsy specimens. These types of changes are more frequently observed in OP developed as a drug adverse reaction.

Despite treatment with cyclophosphamide and radiotherapy (known causes of BOOP), a close relationship between the administration of antibodies and the manifestation of general symptoms and pulmonary infiltrates were observed in this patient, proved by the withdrawal of transtuzumab therapy. The partial regression of pulmonary opacities occurred 2 weeks later and consequently steroid therapy was not administered. Three months after stopping transtuzumab treatment, an improvement in clinical status, a decrease of ESR and a spontaneous regression of pulmonary densities was observed. The authors believe that this is evidence of the connection between OP and transtuzumab treatment. In drug-induced OP it is sometimes difficult to determine a causative factor, since OP may be associated with various drugs. Resolution of OP after stopping the drug is obviously the best clue to establish causality. The probability that transtuzumab was a causal factor of OP is very high. However, in this case other factors such as chemotherapy or radiation may have influenced the development of OP.

Transtuzumab is a humanised monoclonal antibody, which blocks the receptor for epidermal growth factor type 2 (HER 2 ), blocking the stimulation of the proliferation of the neoplastic cells. Transtuzumab is applied in the treatment of breast cancer, when neoplastic cells present overexpression of this receptor (3+). It is an effective and well-tolerated drug [1].

COOK-BRUNS [8] presented the retrospective analysis of the side-effects of transtuzumab in 25,000 patients. Cardiotoxicity was the main side-effect, but it was connected with previous antracyclines treatment. Within a presented group of patients there were 74 serious reactions, including bronchospasm, cardiorespiratory failure, anaphylactic shock, vaso-motor oedema and tachycardia during the first infusion of transtuzumab. Nine of these patients eventually died and had suffered earlier from the ventilatory disorders and respiratory failure due to neoplasm. Moreover, the adult respiratory distress syndrome was diagnosed in some patients. However, OP was not recognised in any of the cases.

This report presents the first incidence of organising pneumonia during transtuzumab treatment. 


\section{References}

1. Nabholtz JM, Slamon D. New adjuvant strategies for breast cancer: meeting the challenge of integrating chemotherapy and transtuzumab (Herceptin). Semin Oncol 2001; 1: Suppl. $3,1-12$.

2. Bayle JY, Nesme P, Bejui-Thivolet F, Loire R, Guerin JC, Cordier JF. Migratory organizing pneumonitis "primed" by radiation therapy. Eur Respir J 1995; 8: 322-326.

3. Crestani B, Valeyre D, Roden S, Wallaert B, Dalphin JC, Cordier JF. Bronchiolitis obliterans organizing pneumonia syndrome primed by radiation therapy to the breast. The Groupe d'Etudes et de Recherche sur les Maladies Orphelines Pulmonaries (GERM"O"P). Am J Respir Dis 1998; 158: $1929-1935$.
4. Lohr RH, Boland BJ, Douglas WW, et al. Organizing pneumonia. Features and prognosis of cryptogenic, secondary, and focal variants. Arch Int Med 1997; 157: 13231329.

5. King TE Jr, Mortenson RL. Cryptogenic organizing pneumonitis. The North American experience. Chest 1992; 102: 8-13.

6. Arbetter KR, Parkash UB, Tazelaar HD, Douglas WW. Radiation-induced pneumonitis in the "nonirradiated" lung. Mayo Clin Proc 1999; 74: 27-36.

7. Costabel U, Teshler H, Schoenfeld B, et al. BOOP in Europe. Chest 1992; 102: 14-20.

8. Cook-Bruns N. Retrospective analysis of the safety of Herceptin immunotherapy in metastatic breast cancer. Oncology 2001; 61: Suppl. 2, 58-66. 